\title{
Low selection rate of HLA-anchor escape mutations in HIV after transmission of subtype B and recombinants BF strains patients from Argentina
}

\author{
G Damilano $^{1 *}$, E Socias², C Magneres², G Turk', M Gomez-Carrillo', H Salomon', D Dilernia ${ }^{1}$
}

From AIDS Vaccine 2012

Boston, MA, USA. 9-12 September 2012

\section{Background}

The immune response of HIV-infected individuals shapes the evolution of the virus by selecting escape mutation. After transmission to a new host, the HLA-mediated immune pressure changes. The objective of our study was to characterize the dynamics of HLA-anchor escape mutations after transmission.

\section{Methods}

We studied 6 transmission events between members of serodiscordant couples collecting blood samples from the donor and the previously seronegative-partner at the moment of seroconversion, and a second sample at least 6 months post-infection. HLA-I typing was performed by the SSOP-PCR method. The viral gene gag was amplified by RT-PCR and cloned into the pGEM-T vector for viral quasiespecies analysis. Transmitted strain was identified by phylogenetic analysis. Escape mutation was defined as viral polimorphisms located in HLA-anchor position that eliminate an epitope predicted by the NetMHC (CBS Prediction Server). Significant variations in the number of escape mutations were assessed by Poisson's probability distribution.

\section{Results}

Of the total epitopes available in transmitted gag for recognition by the recipient HLA alleles, a mean $7.25 \%$ of them selected escape mutations by the moment of the second sample collection in an anchor position of the epítope. Of the total of HLA-anchor escape mutations present in the second sample and absent in the subtype consensus, a mean $79,8 \%$ were already present in the first sample of the recipient and in the sample of the donors. Also, the majority (84\%) of the escape mutations to the donor's HLA alleles persisted in time after transmission without reversion, even in the absence of the selecting HLA allele.

\section{Conclusion}

The low rate of newly selected escape mutations could be due in part to lack of immune pressure, but considering the high rate of transmission and persistence, our results suggest a high level of viral adaptation to the HLA system in subtype B and recombinants BF circulating in Argentina.

\section{Author details}

${ }^{1}$ Argentinean Reference Center for AIDS, Buenos Aires, Argentina. ${ }^{2}$ HUESPED Foundation, Buenos Aires, Argentina.

Published: 13 September 2012

doi:10.1186/1742-4690-9-S2-P140

Cite this article as: Damilano et al:: Low selection rate of HLA-anchor escape mutations in HIV after transmission of subtype $B$ and recombinants BF strains patients from Argentina. Retrovirology 2012 9(Suppl 2):P140. 